\title{
ARTICLE
}

Pediatrics

\section{Genetic predisposition to higher body fat yet lower cardiometabolic risk in children and adolescents}

\author{
Anna Viitasalo ${ }^{1}$ Theresia M. Schnurr $\mathbb{D}^{1}$ - Niina Pitkänen $\mathbb{D}^{2} \cdot$ Mette Hollensted $^{1} \cdot$ Tenna R. H. Nielsen $^{3,4}$. \\ Katja Pahkala ${ }^{2,5} \cdot$ Niina Lintu $^{6} \cdot$ Mads V. Lind $\mathbb{D}^{7} \cdot$ Mustafa Atalay $^{6} \cdot$ Christine Frithioff-Bøjsøe $^{1,3}$. \\ Cilius E. Fonvig $\mathbb{D}^{1,3,8} \cdot$ Niels Grarup $\mathbb{1}^{1} \cdot$ Mika Kähönen $^{9,10} \cdot$ Anni Larnkjaer ${ }^{7}$ - Oluf Pedersen $\mathbb{D}^{1}$. \\ Jens-Christian Holm ${ }^{1,3,11} \cdot$ Kim F. Michaelsen $^{7} \cdot$ Timo A. Lakka $^{6,12,13} \cdot$ Terho Lehtimäki $^{10,14} \cdot$ Olli Raitakari $^{2,15}$. \\ Torben Hansen ${ }^{1} \cdot$ Tuomas O. Kilpeläinen ${ }^{1}$
}

Received: 30 July 2018 / Revised: 6 June 2019 / Accepted: 8 June 2019 / Published online: 22 July 2019

(c) The Author(s), under exclusive licence to Springer Nature Limited 2019

\begin{abstract}
Background Most obese children show cardiometabolic impairments, such as insulin resistance, dyslipidemia, and hypertension. Yet some obese children retain a normal cardiometabolic profile. The mechanisms underlying this variability remain largely unknown. We examined whether genetic loci associated with increased insulin sensitivity and relatively higher fat storage on the hip than on the waist in adults are associated with a normal cardiometabolic profile despite higher adiposity in children.

Methods We constructed a genetic score using variants previously linked to increased insulin sensitivity and/or decreased waist-hip ratio adjusted for body mass index (BMI), and examined the associations of this genetic score with adiposity and cardiometabolic impairments in a meta-analysis of six cohorts, including 7391 European children aged 3-18 years.

Results The genetic score was significantly associated with increased degree of obesity (higher BMI-SDS beta $=0.009 \mathrm{SD} /$ allele, $\mathrm{SE}=0.003, P=0.003$; higher body fat mass beta $=0.009, \mathrm{SE}=0.004, P=0.031$ ), yet improved body fat distribution (lower $\mathrm{WHR}_{\text {adjBMI }}$ beta $=-0.014 \mathrm{SD} /$ allele, $\mathrm{SE}=0.006, P=0.016$ ), and favorable concentrations of blood lipids (higher HDL cholesterol: beta $=0.010 \mathrm{SD} /$ allele, $\mathrm{SE}=0.003, P=0.002$; lower triglycerides: beta $=-0.011 \mathrm{SD} /$ allele, $\mathrm{SE}$ $=0.003, P=0.001$ ) adjusted for age, sex, and puberty. No differences were detected between prepubertal and pubertal/ postpubertal children. The genetic score predicted a normal cardiometabolic profile, defined by the presence of normal glucose and lipid concentrations, among obese children ( $\mathrm{OR}=1.07 \mathrm{CI} 95 \% 1.01-1.13, P=0.012, n=536)$.

Conclusions Genetic predisposition to higher body fat yet lower cardiometabolic risk exerts its influence before puberty.
\end{abstract}

\section{Introduction}

The prevalence of pediatric overweight and obesity has increased worldwide during the past decades [1]. Most

These authors contributed equally: Anna Viitasalo and Theresia M. Schnurr

Supplementary information The online version of this article (https:// doi.org/10.1038/s41366-019-0414-0) contains supplementary material, which is available to authorized users.

Anna Viitasalo

anna.viitasalo@uef.fi

Extended author information available on the last page of the article overweight or obese children exhibit cardiometabolic risk factors, such as insulin resistance, impaired glucose tolerance, dyslipidemia, and elevated blood pressure [2]. However, depending on the criteria used, $3-68 \%$ of obese children and adolescents have been found to have a cardiometabolic risk profile within normal range, a controversial condition sometimes called "metabolically healthy obesity" [3]. While the clinical usefulness and stability of this condition have been questioned, these observations suggest that the effect of body adiposity on cardiometabolic health may vary among children and adolescents [3]. The mechanisms underlying such differences remain largely unknown.

In adult populations, many genetic variants associated with increased insulin sensitivity [4] and relatively higher fat storage on the hip than on the waist [5], are related to increased body fatness, yet improved cardiometabolic risk 
profile. These findings may reflect an enhanced ability to store fat subcutaneously, which may lead to a decreased accumulation of ectopic fat and prevention of lipotoxic effects $[6,7]$. While it remains unclear whether such effects are already apparent in childhood, longitudinal studies suggest that some obese children with a favorable metabolic profile may preserve the phenotype into adulthood [8]. This indicates that the underlying mechanisms may be partly shared between children and adults. Identification of genetic variation contributing to the link between adiposity and its complications in children and adolescents is important, as it could shed light on the underlying mechanisms and help distinguishing between the children who are most and least prone to developing cardiometabolic impairments upon weight gain.

Here, we report the results of a meta-analysis of 7391 children and adolescents from Finland, Denmark, and the United Kingdom, showing that genetic predisposition to increased body fat yet improved metabolic profile is observed in both prepubertal and postpubertal children and adolescents, and is associated with higher odds of having normal glucose and lipid concentrations despite obesity.

\section{Materials/subjects and methods}

\section{Study populations}

The present study includes 2970 adolescents $16-18$ years of age from the Avon Longitudinal Study of Parents and Children (ALSPAC) [9]; 2099 Finnish children and adolescents 3-18 years of age from the Cardiovascular risk in Young Finns Study (YFS) [10]; 705 Danish overweight or obese children and adolescents aged 3-18 years from The Danish Childhood Obesity Biobank (TDCOB cases) as well as a population-based control sample comprising 361 Danish children and adolescents (TDCOB controls) 6-18 years of age [11]; 470 Finnish adolescents 14-15 years of age from the Special Turku Coronary Risk Factor Intervention Project (STRIP) [12]; 460 Finnish children 6-9 years of age from the Physical Activity and Nutrition in Children (PANIC) study [13]; and 326 Danish children 3 years of age from the Småbørns Kost Og Trivsel I and II (SKOT) studies [14]. We excluded children without genetic data or body mass index (BMI), and children with known history of type 1 diabetes ( 31 children), type 2 diabetes ( 2 children), mental or developmental disorders (29 children), or known monogenic forms of obesity (21 children). We also excluded children with non-European ancestry or with known medication for hypercholesterolemia or hypertension. For twin-pairs, one twin was excluded. All studies were conducted in accordance with the principles of the Declaration of Helsinki and were accepted by the local research ethic committees. A written informed consent was obtained from all parents/children in all studies. Details of the participating studies are provided in Supplementary Material.

\section{Measurements of body size and composition, cardiometabolic risk factors, and puberty status}

Body height and body weight were measured in all studies, and BMI was calculated as body weight $(\mathrm{kg})$ divided by height squared $\left(\mathrm{m}^{2}\right)$. BMI-SDS was calculated according to Finnish (PANIC, STRIP, and YFS) [15], Danish (SKOT, TDCOB cases, and TDCOB controls) [16], and UK (ALSPAC) [17] national reference values. Waist circumference was measured at mid-distance between the bottom of the rib cage and the top of the iliac crest. Hip circumference was measured at the level of the greater trochanters. Body fat mass, body lean mass, and body fat percentage were measured using bio impedance analysis (STRIP and SKOT) or dual-energy X-ray absorptiometry (PANIC, ALSPAC, and TDCOB). Blood pressure was measured manually using calibrated sphygmomanometers (PANIC and YFS) or an oscillometric device (ALSPAC, TDCOB, STRIP, and SKOT). Blood samples were taken after overnight fast in ALSPAC, YFS, TDCOB, STRIP, and PANIC studies and after $>2 \mathrm{~h}$ fasting in SKOT. Plasma glucose was measured using the hexokinase method. Serum insulin was analyzed by an ultrasensitive ELISA automated microparticle enzyme immunoassay in the ALSPAC study, a coated charcoal immunoassay in the YFS study, an electrochemiluminescent immunoassay in the TDCOB and PANIC studies, a microparticle enzyme immunoassay in the STRIP study, and by a chemiluminescent immunometric assay in the SKOT study. Triglycerides, total, LDL, and HDL cholesterol were measured enzymatically. Overweight and obesity were defined using the age and sex-specific BMI cutoffs of the International Obesity Task Force (IOTF) [18]. In YFS, TDCOB cases, STRIP, and PANIC, the research physician or study nurse assessed pubertal status using the 5-stage criteria described by Tanner [19, 20]. In TDCOB controls, puberty was self-evaluated using picture charts. The boys were defined as having entered clinical puberty if their testicular volume assessed by an orchidometer was $>3 \mathrm{ml}$ (stage $\geq 2$ ). The girls were defined as having entered clinical puberty if their breast development had started (stage $\geq 2$ ). To divide children into preonset and onset/postonset groups, children with stage 1 in the Tanner scale were considered preonset and all others were considered onset/postonset. Children in the SKOT study (aged 3 years) were all considered preonset and children in the ALSPAC study (aged 17 years) were all considered onset/ postonset. The assessments have been previously described in detail for each study population [12, 21-25]. 


\section{Genotyping and imputation}

Children in YFS, TDCOB, and SKOT were genotyped using the Illumina Infinium HumanCoreExome BeadChip (Illumina, San Diego, CA, USA). Children in STRIP were genotyped using the Illumina HumanCardio-Metabo BeadChip. Children in PANIC were genotyped using both Illumina Infinium HumanCoreExome Beadchip and Illumina Infinium Cardio-Metabo Beadchip, and the genotypes from the two arrays were combined. Children in ALSPAC were genotyped using the Illumina HumanHap550 Quad chip. In all studies, genotype imputations were performed using the 1000 Genomes European-ancestry reference panel.

To construct genetic scores, we used 53 single nucleotide polymorphisms (SNPs) previously reported to associate with an insulin resistance-related phenotype (defined as higher fasting insulin concentrations adjusted for BMI, higher triglyceride concentrations, and lower concentration of HDL cholesterol) [4] in adults, and 49 SNPs reported to associate with waist-hip ratio adjusted for BMI $\left(\mathrm{WHR}_{\text {adjBMI }}\right)$ in a genome-wide association study (GWAS) of adult population [5]. Genetic scores were calculated as the sum of insulin resistance phenotype-increasing alleles or WHRadjBMI-increasing alleles, respectively. For imputed genotypes, the genetic score construction was based on genotype dosages.

\section{Statistical methods}

All analyses were performed using $\mathrm{R}$ (version 3.3.1). We explored the adiposity-increasing effect of the examined genetic scores in children using the R package 'gtx' [26] to examine associations of insulin resistance- and $\mathrm{WHR}_{\text {adjBMI}}{ }^{-}$ decreasing genetic scores with increased BMI in the largest publicly available childhood BMI GWAS meta-analysis summary data contributed by the Early Growth Genetics (EGG) Consortium and downloaded from www.eggconsortium.org [27]. Linear regression models for inverse normally transformed residuals, adjusted for age, sex, puberty, study group if needed (for SKOT and STRIP), and the first three genome-wide principal components, were used to examine the associations of the genetic score with the degree of obesity and cardiometabolic variables in each participating study population. For waist circumference, WHR, and adiponectin, we additionally adjusted the residuals for BMI. For systolic and diastolic blood pressure, we additionally adjusted the residuals for height. The effect sizes are reported in standard deviations (SD) of the inverse normally transformed traits (mean $=0, \mathrm{SD}=1$ ). We also performed analyses stratified by weight status (normal weight vs. overweight/obese), puberty (preonset vs. onset/ postonset), and sex. Independent samples $t$-test was used to compare the differences on the effect of the genetic score for cardiometabolic variables in the stratified analyses between groups. Logistic regression models adjusted for age, sex, puberty, and the first three genome-wide principal components were used to study the association of the genetic score with a normal cardiometabolic profile among overweight and obese children and adolescents. The results from the different studies were pooled by fixed effects metaanalyses using the 'meta' package in R (version 4.6.0) [28].

\section{Results}

\section{Basic characteristics}

Of the 7391 children and adolescents included in the six participating study populations, 2405 (33\%) were prepubertal according to Tanner stage, and 1685 (23\%) were overweight or obese (Table 1). The mean age of the children and adolescents was 13.1 years (range 2.7-18.0 years).

\section{Construction of the adiposity-increasing genetic score with cardiometabolically protective effects}

For some genetic variants, the allele known to associate with decreased insulin resistance [4] or waist-hip ratio [5] is related to increased BMI in adult populations. However, alleles in some variants do not show a BMI-increasing effect and thus may impact insulin resistance through other mechanisms than that of subcutaneous fat expandability. Furthermore, some genetic variants exert varying effect sizes during a life span [29]. To identify the insulin resistance and $\mathrm{WHR}_{\text {adjBMI }}$ associated variants that may have an adiposity increasing yet a cardiometabolically protective effect in children and adolescents, we first screened the 53 known insulin resistance-decreasing and the 49 known WHR $_{\text {adjBMI-decreasing variants for their effects on adult }}$ BMI in the summary data reported in the supplemental material of the respective GWAS analyses in adults $[4,5]$ and associated variants were then tested for their effects on childhood BMI in data from the EGG Consortium $(n=35$ 665) [27].

We found that 30 of the 53 known adult insulin resistance-decreasing variants, and 38 of the 49 known adult WHR $_{\text {adjBMI }}$-decreasing variants, displayed a BMI-increasing direction of effect in adults (Supplementary Tables 1-2). Of these, 18 insulin resistance and $23 \mathrm{WHR}_{\text {adjBMI-associated }}$ variants showed a nominally significant $(P<0.05)$ association with adult BMI. We constructed a score for each of these four groups of loci, i.e. including 30 or 18 insulin resistance variants and 38 or $23 \mathrm{WHR}_{\text {adjBMI }}$ variants, to test for associations with childhood BMI in the summary results of the EGG Consortium. We found that the scores comprising 18 insulin resistance-decreasing variants and 23 
Table 1 Characteristics of children and adolescents in the studies included in the present meta-analyses

\begin{tabular}{|c|c|c|c|c|c|c|c|}
\hline & ALSPAC & YFS & TDCOB cases & TDCOB controls & STRIP & PANIC & SKOT \\
\hline$N$ (total) & 2970 & 2099 & 705 & 361 & 470 & 460 & 326 \\
\hline Girls (\%) & $1637(55 \%)$ & $1139(54 \%)$ & $415(59 \%)$ & $238(66 \%)$ & $227(48 \%)$ & $219(48 \%)$ & $154(47 \%)$ \\
\hline Prepubertal (\%) & $0(0 \%)$ & $1244(51 \%)$ & $314(45 \%)$ & $73(22 \%)$ & $0(0 \%)$ & $448(97 \%)$ & $326(100 \%)$ \\
\hline Overweight/obese $^{a}$ & $634(21 \%)$ & $162(8 \%)$ & $699(99 \%)$ & $46(13 \%)$ & $54(12 \%)$ & $56(12 \%)$ & $34(10 \%)$ \\
\hline Age (years) & $17.6(0.2)$ & $9.8(4.0)$ & $11.5(2.9)$ & $13.0(3.1)$ & $15.0(0.0)$ & $7.6(0.4)$ & $3.0(0.1)$ \\
\hline Body height $(\mathrm{cm})$ & $171(9)$ & $137(25)$ & $152(16)$ & 157 (16) & $170(8)$ & $129(6)$ & $96.2(3.6)$ \\
\hline Body weight (kg) & $66.8(13.5)$ & $35.1(16.5)$ & $64.9(23.9)$ & $48.4(15.3)$ & $61.3(6.9)$ & $26.7(4.8)$ & $14.9(1.7)$ \\
\hline BMI $\left(\mathrm{kg} / \mathrm{cm}^{2}\right)$ & $22.7(4.0)$ & $17.4(2.8)$ & $27.0(5.3)$ & $19.1(3.2)$ & $20.5(3.3)$ & $16.1(2.0)$ & $16.1(1.2)$ \\
\hline BMI-SDS & $0.37(1.16)$ & $-0.29(1.00)$ & $2.90(0.66)$ & $0.31(1.05)$ & $-0.08(0.97)$ & $-0.20(1.1)$ & $0.43(0.92)$ \\
\hline Waist circumference $(\mathrm{cm})$ & NA & NA & $93(15)$ & $70(9)$ & $73(8)$ & $57(5)$ & $47(4)$ \\
\hline Waist-hip-ratio & NA & NA & $0.97(0.07)$ & $0.82(0.1)$ & $0.80(0.05)$ & $0.85(0.0)$ & NA \\
\hline Total body lean mass (kg) & $45.7(10.0)$ & NA & NA & NA & $45(9)$ & $21(2)$ & NA \\
\hline Total body fat mass $(\mathrm{kg})$ & $17.9(10.2)$ & NA & $28.0(12.2)$ & NA & $12.7(7.5)$ & $5.6(3.3)$ & $2.6(0.8)$ \\
\hline Body fat percentage $(\%)$ & $25.3(11.5)$ & NA & $43.6(5.2)$ & NA & $20.9(9.3)$ & $20(8)$ & $17.4(4.3)$ \\
\hline Adiponectin $(\mu \mathrm{g} / \mathrm{ml})$ & NA & $13.7(5.5)$ & $4.2(2.4)$ & $5.7(4.8)$ & $10.4(4.4)$ & $9.1(4.2)$ & NA \\
\hline Insulin (mU/l) & $8.1(7.7)$ & $9.2(5.8)$ & $6.9(7.2)$ & $4.5(2.2)$ & $8.3(3.5)$ & $4.5(2.5)$ & $3.2(3.5)$ \\
\hline Glucose (mmol/l) & $5.0(0.4)$ & NA & $5.2(0.6)$ & $5.4(1.1)$ & $4.9(0.3)$ & $4.8(0.4)$ & $4.8(0.6)$ \\
\hline LDL cholesterol (mmol/l) & $2.1(0.6)$ & $3.5(0.8)$ & $2.5(0.8)$ & $2.2(0.5)$ & $2.4(0.7)$ & $2.3(0.5)$ & $2.5(0.6)$ \\
\hline HDL cholesterol (mmol/l) & $1.3(0.3)$ & $1.6(0.3)$ & $1.2(0.3)$ & $1.5(0.3)$ & $1.2(0.2)$ & $1.6(0.3)$ & $1.2(0.2)$ \\
\hline Triglycerides (mmol/l) & $0.83(0.36)$ & $0.65(0.29)$ & $1.1(0.6)$ & $0.7(0.3)$ & $0.85(0.42)$ & $0.60(0.25)$ & $1.1(0.6)$ \\
\hline Systolic blood pressure (mmHg) & $114.3(9.6)$ & $111(12)$ & $114(12)$ & $114(10)$ & $117(12)$ & $100(7)$ & $96(8)$ \\
\hline Diastolic blood pressure (mmHg) & $64.0(6.2)$ & $68(9)$ & $65(8)$ & $62(7)$ & $61(9)$ & $61(7)$ & $61(7)$ \\
\hline
\end{tabular}

The values are expressed as means (standard deviations)

BMI-SDS BMI standad deviation score [15-17], GRS genetic score, IR insulin resistance, WHRadjBMI waist-hip ratio adjusted for BMI

${ }^{a}$ Overweight and obesity were defined using the age- and sex-specific BMI cutoffs of the International Obesity Task Force (IOTF) criteria [18]

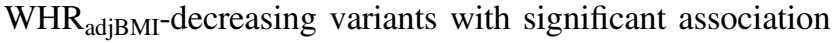
with increased BMI in adults, exhibited more pronounced associations with BMI in children (beta $=0.007 \mathrm{SD} /$ allele, $\mathrm{SE}=0.002, \quad P=2.6 \times 10^{-5}$; and beta $=0.009 \mathrm{SD} /$ allele, $\mathrm{SE}=0.002, P=9.7 \times 10^{-7}$, respectively) as compared to the scores including 30 insulin sensitivity (beta $=0.006 \mathrm{SD} /$ allele, $\mathrm{SE}=0.002, P=0.0002$ ) and $38 \mathrm{WHR}_{\mathrm{adjBMI}}$ variants (beta $=0.006 \mathrm{SD} /$ allele, $\mathrm{SE}=0.001$, and $P=8.1 \times 10^{-5}$ ). Finally, we constructed a combined genetic score of these 18 insulin resistance and $23 \mathrm{WHR}_{\mathrm{adjBMI}}$-decreasing loci. We excluded seven variants that were in linkage disequilibrium with each other $\left(r^{2}>0.1\right)$ by excluding the variant with weaker association (higher $p$-value) with BMI in the summary results of EGG-Consortium data. One of the WHR $_{\text {adjBMI-reducing SNPs, rs7759742, was not available in }}$ all six participating studies of the present meta-analyses, however, and was therefore excluded from the final genetic score. The combined score comprising 33 independent variants displayed the strongest association with increased $\mathrm{BMI}$ in the EGG-Consortium data (beta $=0.009 \mathrm{SD} /$ allele, $\mathrm{SE}=0.002, P=3.2 \times 10^{-9}$ ) and was taken forward to test for associations with adiposity and cardiometabolic profiles in a meta-analysis of the six participating cohorts.

\section{Association of the genetic score with adiposity and cardiometabolic traits in children and adolescents}

In a meta-analysis of all 7391 children and adolescents from the six participating studies, the combined score of the 33 independent insulin resistance and/or $\mathrm{WHR}_{\text {adjBMI-decreas- }}$ ing variants was associated with a higher degree of obesity (BMI-SDS: beta $=0.009 \mathrm{SD} /$ allele, $\mathrm{SE}=0.003, P=0.003$; body fat mass: beta $=0.009 \mathrm{SD} /$ allele, $\mathrm{SE}=0.004, P=$ 0.031 ), yet it had beneficial effects on several cardiometabolic traits, including improved body fat distribution (lower WHR $_{\text {adjвмI }}$ : beta $=-0.014$ SD/allele, $\mathrm{SE}=0.006, \quad P=$ $0.016)$ and favorable concentrations of blood lipids (higher HDL cholesterol: beta $=0.010$ SD/allele, $\mathrm{SE}=0.003, P=$ 0.002; lower triglycerides: beta $=-0.011 \mathrm{SD} /$ allele, $\mathrm{SE}=$ $0.003, P=0.001)$. This genetic score was also associated with higher circulating concentration of adiponectin (beta $=0.020$ SD/allele, $\mathrm{SE}=0.005, P=3 \times 10^{-5}$ ) (Fig. 2, Supplementary Table 3, Supplementary Fig. 1). The results remained similar after excluding the SKOT cohort of 3year-old children (Supplementary Table 4).

Recently, an analysis comparing the obese and population-based samples of the TDCOB study found that 
Fig. 1 Flow chart of the study protocol
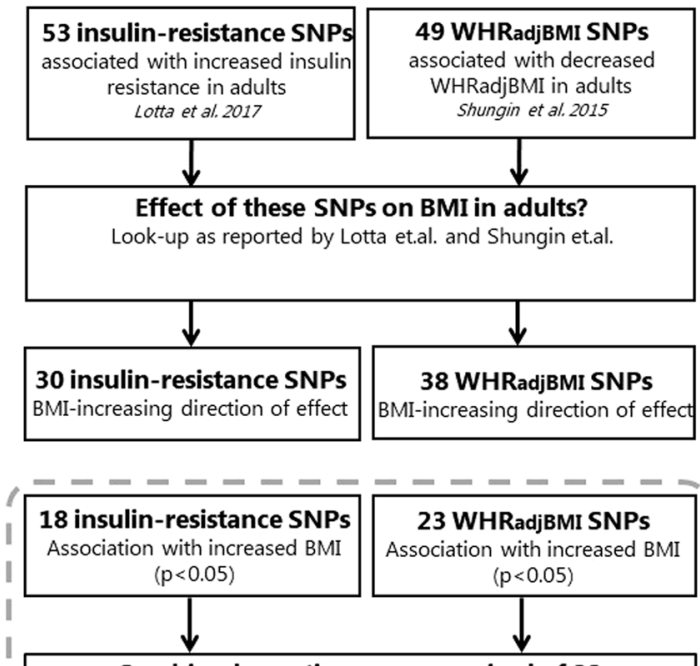
Association with increased BMI $(p<0.05)$

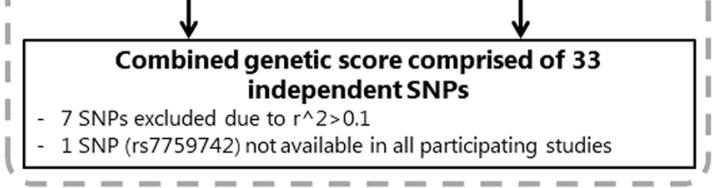

I Most pronounced effects on increased BMI in children

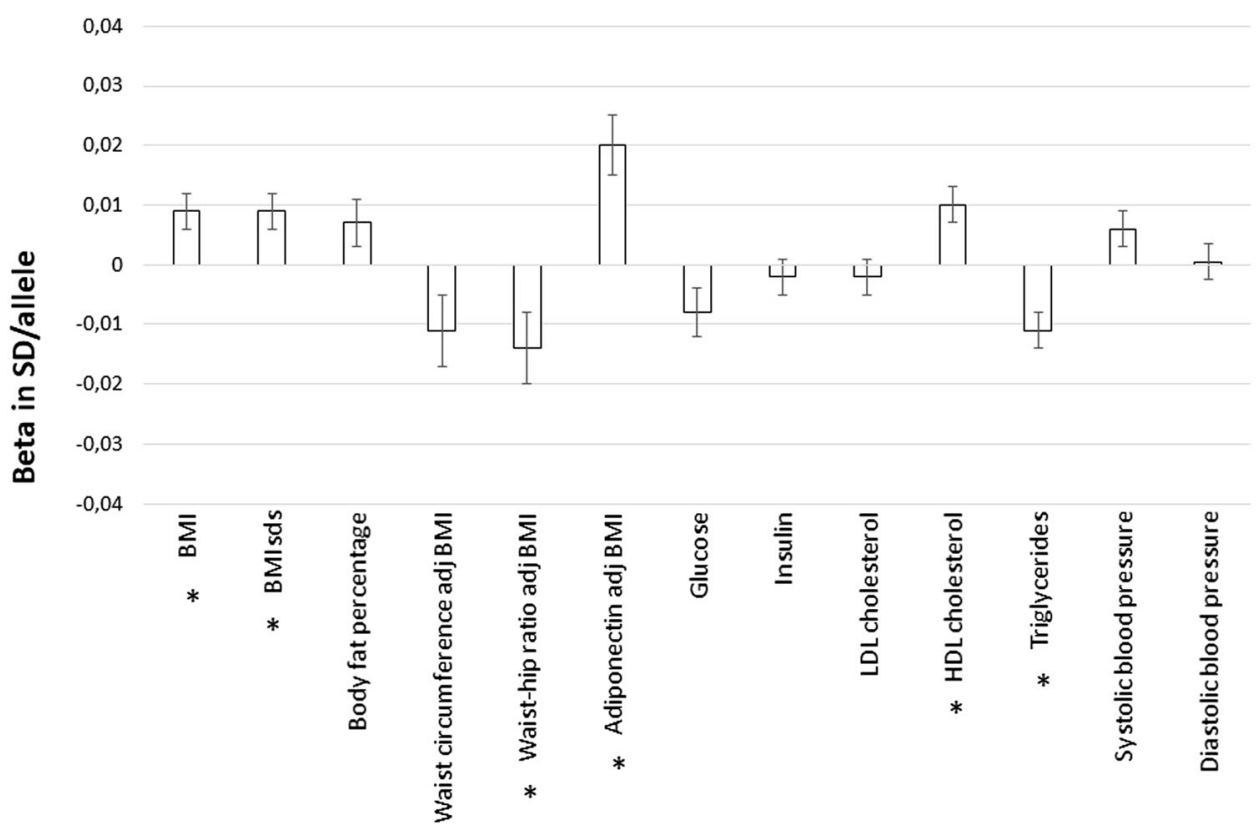

Fig. 2 Linear regression analysis to test the association of the combined insulin sensitivity-increasing and $\mathrm{WHR}_{\mathrm{adjBMI}}{ }^{-}$-decreasing genetic score with adiposity and cardiometabolic variables in all children and adolescents as beta values (standard errors) of the inverse normally transformed traits. The results are aligned according to the insulin sensitivity-increasing/WHR $\mathrm{WdjBM}^{-}$-decreasing allele of the genetic score.

the effects of insulin resistance variants on cardiometabolic traits may be more pronounced in overweight or obese children and adolescents [30]. To examine differences in genetic effects between overweight/obese children and normal weight children in the present meta-analyses, we performed stratified analyses. The combined score of 33
All analyses are adjusted for age, sex, puberty, and first three genomewide principal components. The effects were pooled using fixed effects models meta-analysis. ${ }^{*} P$-values $<0.05$. The numerical values for betas, standard errors, $p$-values, and the number of subjects are presented in Supplementary Table 3

insulin resistance and/or $\mathrm{WHR}_{\mathrm{adjBMI}}$-decreasing variants had a stronger beneficial effect on insulin concentration in the overweight/obese group than in the normal weight group $\left(\mathrm{P}_{\text {DIFFERENCE }}=0.024\right)$ (Supplementary Tables 5-6, Fig. 3), whereas no significant differences between the groups were detected for other cardiometabolic traits. 


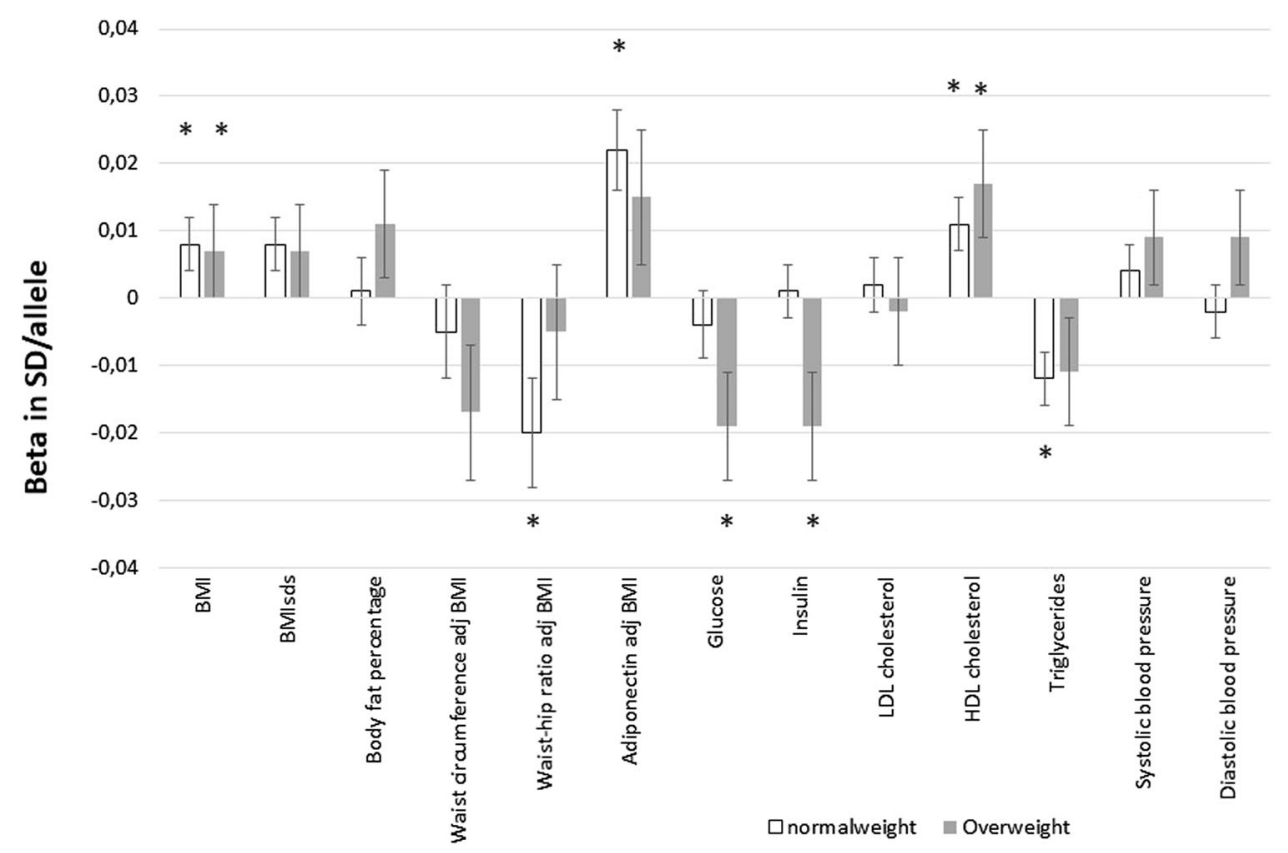

Fig. 3 Linear regression analysis to test the association of the combined insulin sensitivity-increasing and $\mathrm{WHR}_{\text {adjBMI-decreasing genetic }}$ score with adiposity and cardiometabolic variables in normal weight (white column) and overweight/obese (black column) children and adolescents as beta values (standard errors) of the inverse normally transformed traits. The results are aligned according to the insulin

When stratifying the analyses according to pubertal status, we found that the associations of the combined score with adiposity and cardiometabolic traits were consistent between prepubertal (Supplementary Table 7) and pubertal/ postpubertal (Supplementary Table 8) children and adolescents (Supplementary Fig. 2), suggesting that genetic predisposition to increased body fat yet improved cardiometabolic profile exerts its influence already before puberty.

When comparing the effect of the combined score between girls (Supplementary Table 9) and boys (Supplementary Table 10), no significant differences between the groups were detected (Supplementary Fig. 3).

\section{Stratified analyses in children and adolescents with the highest and lowest genetic predisposition}

To quantify the impact of the genetic score in the upper and lower extremes of genetic predisposition, we compared adiposity and cardiometabolic variables in the highest and lowest $10 \%$ of children and adolescents according to the combined genetic score of insulin resistance and/or $\mathrm{WHR}_{\text {adjBMI}}$-decreasing variants. We found that children and adolescents in the highest $10 \%$ of the genetic score had $0.27 \mathrm{~kg} / \mathrm{m}^{2}$ higher BMI $(P<0.001), 0.10$ unit higher BMISDS $(P=0.009), 0.01$ unit lower $\mathrm{WHR}_{\text {adjBMI }}(P=0.035)$,
sensitivity-increasing/WHR ${ }_{\mathrm{adjBM}}$-decreasing allele of the genetic score. All analyses are adjusted for age, sex, puberty, and first three genomewide principal components. The effects were pooled using fixed effects models meta-analysis. ${ }^{*} P$-values $<0.05$. The numerical values for betas, standard errors, $p$-values, and the number of subjects are presented in Supplementary Tables 5-6

$0.07 \mathrm{mmol} / \mathrm{l}$ lower triglycerides $(p=0.001)$, and $0.99 \mu \mathrm{g} / \mathrm{ml}$ higher adiponectin concentrations $(P<0.001)$ than children and adolescents in the lowest $10 \%$ of the score. There were no differences in other variables tested.

\section{Association of the genetic score with a normal cardiometabolic profile in overweight and obese children and adolescents}

Previous studies among children and adolescents have used various criteria to define "metabolically healthy obesity" [3]. We dichotomized obese children and adolescents as having either a cardiometabolically normal or unhealthy profile according to the presence of elevated glucose and triglyceride concentrations, decreased concentration of HDL cholesterol, and elevated systolic and/or diastolic blood pressure. The age-specific adolescent metabolic syndrome criteria that are linked to the health-based Adult Treatment Panel III (ATP) and International Diabetes Federation adult criteria [31] were used to define cutoffs for individuals 12 years or older, whereas criteria based on the modified ATP III MS definition [32] were applied for those aged below 12 years [33]. We used two different definitions for cardiometabolic health; one that included elevated blood pressure in its definition, and one that did not. Of the 536 obese children and adolescents participating in the TDCOB 
cases and ALSPAC studies, $36 \%$ and $24 \%$ were defined as having cardiometabolically normal profile based on a definition excluding and including blood pressure, respectively. The combined score predicted the absence of cardiometabolic impairments when blood pressure was not included the criteria $(\mathrm{OR}=1.07 \mathrm{CI} 95 \% 1.01-1.13, P=0.012, n=$ $536)$, yet not when blood pressure was included (OR $=1.02$ CI $95 \% 0.97-1.08, P=0.445, n=509)$. We also tested whether the combined score predicted a normal cardiometabolic profile in overweight and obese children combined. Among the 1023 children who had data available on systolic and/or diastolic blood pressure, and fasting levels of glucose, triglycerides, and HDL cholesterol, there was a significant association with a normal cardiometabolic profile when blood pressure was not included in the criteria (OR $=1.04,95 \%$ CI 1.01-1.07, $P=0.018, n=1081)$, yet not when blood pressure was included $(\mathrm{OR}=1.02,95 \% \mathrm{CI}$ $0.99-1.06, P=0.239 n=1023)$.

\section{Discussion}

The results of this large meta-analysis indicate that genetic predisposition increased insulin sensitivity and relatively higher fat storage on the hip compared to the waist might lead to increased body fat yet improved cardiometabolic risk profile in children and adolescents. The associations were comparable between prepubertal and pubertal/postpubertal children and adolescents, suggesting that genetic susceptibility to cardiometabolically normal profile in obesity is expressed before puberty. The protective effect of the genetic score on insulin concentrations was more pronounced among overweight/obese children than among normal weight children, suggesting that these genetic effects might be accentuated by excess weight gain. We also showed that the genetic score predicts a cardiometabolically normal status, defined by the presence of normal glucose, triglyceride, and HDL cholesterol concentrations in overweight and obese children and adolescents.

Our findings may reflect a beneficial impact of the genetic score on the ability to store fat subcutaneously rather than viscerally or otherwise ectopically, which has been suggested to be an underlying mechanism for both insulin resistance and $\mathrm{WHR}_{\text {adjBMI }}$ loci $[4,5]$. Subcutaneous fat tissue is the naturally preferred place to store lipids, and when its capacity becomes saturated, the excess fat may "over spill" to non-adipose tissues [34]. The excess of lipids may then accumulate in metabolically relevant organs such as pancreatic beta cells, liver, heart, and skeletal muscle, where they may lead to lipotoxic effects.

Given that increased muscle mass has a favorable effect on cardiometabolic health, we also studied whether the association of the genetic score with increased BMI could be due to increased muscle mass. However, the genetic score showed strong association with increased body fat mass but no association with body lean mass, which suggests that the underlying genetic mechanisms are mainly related to adipose tissue $[4,5]$. Aerobic fitness has a beneficial impact on cardiometabolic health, independent of body adiposity. While it remains to be examined whether the genetic score is associated with aerobic fitness, such association seems unlikely considering the adipose-related effect of this score.

Our results underline that some children and adolescents may be genetically more resistant to cardiometabolic impairments despite higher body fat. On the flipside of the same coin, children and adolescents carrying the opposite alleles, i.e. insulin resistance and $\mathrm{WHR}_{\text {adjBMI }}$-increasing alleles, may be predisposed to cardiometabolic impairments despite a leaner phenotype. Indeed, our stratified analyses indicated that children and adolescents in the lowest decile of our genetic score were leaner but displayed impaired fat distribution, elevated concentrations of triglycerides, and lower concentrations of adiponectin than children and adolescents in the highest decile. This highlights the importance of a healthy lifestyle, also among lean children and adolescents, as some of these children and adolescents might be particularly susceptible to metabolic impairments upon weight gain. Vice versa, some studies suggest that individuals with obesity yet a cardiometabolically healthy profile may not be able to significantly reduce their cardiometabolic risk with antiobesity treatment strategies $[3,35,36]$. Thus, there is a need to better understand obesity-related cardiometabolic impairments in order to improve the effectiveness of measures taken to prevent the cardiometabolic comorbidities of obesity.

In contrast to other cardiometabolic variables, we found that the adiposity-increasing genetic score we examined was not associated with beneficial effects on blood pressure. In contrast, we found an association with increased blood pressure, suggesting that biological mechanisms regulating the link between increased body fatness and elevated blood pressure may be distinct from those regulating the relationship between body fatness and other cardiometabolic risk factors. Indeed, body fatness is suggested to impact blood pressure largely through mechanical stress and chronic over activation of the sympathetic nervous system, acting independently from the pathways regulating insulin resistance and dyslipidemia [37]. We also found that the adiposity-increasing genetic score predicted the absence of cardiometabolic impairments only when blood pressure was excluded from the criteria. Thus, it may be important to consider hypertension separately from other cardiometabolic risk factors when evaluating children's need for treatment interventions. 
Puberty is a time of considerable metabolic and hormonal changes and is associated with a marked decrease in insulin sensitivity [38]. It has been reported that obese adolescents do not sustain insulin sensitivity at the end of puberty [39]. Therefore, the stability of cardiometabolically normal profile among obese children in puberty has been questioned and entering puberty has been considered as a predictor for switching from "metabolically healthy" to unhealthy obese state [39]. In the present study, we found that the adiposity-increasing yet metabolically beneficial effects of the genetic score were found independent of pubertal status, suggesting that the underlying biological mechanisms may be functioning already before puberty. As the adiposity increasing yet cardiometabolically protective genetic effects may start before puberty, their impact on cardiometabolic risk during the life span may be considerable.

The development of cardiometabolic disorders depends on the duration and degree of obesity. Longitudinal studies are warranted to investigate if the genetic effects predisposing to cardiometabolically normal profile despite obesity remain stable over time and upon additional weight gain. Regardless of the protective role of these particular genetic effects on cardiometabolic risk, obesity is never a benign condition as it is associated also with an increased risk of a number of other harmful conditions, such as sleep apnea, certain cancers, and psychosocial problems [2].

We applied genetic scores, a commonly used tool in genetic analysis [40], to robustly estimate genetic predisposition to increased insulin sensitivity and relatively higher fat storage on the hip compared to the waist and interpreted our results assuming that the genetic loci included in the genetic scores have a unified and unidirectional effect on insulin sensitivity or $\mathrm{WHR}_{\mathrm{adjBMI}}$, respectively, and show a unison direction of effect on adiposity. However, this may not hold true for all variants included in the genetic scores in the present study and likely, the genetic scores are resembling an average of the effect of associated and null loci that may or may not be working in the same direction across phenotypes. Therefore, the results of our association analyses do not allow us to draw conclusions about underlying biological factors but instead provide new hints to understand the genetic architecture behind the cardiometabolically normal phenotype in obese children and adolescents and hold promise for targeted treatment strategies that could diminish the cardiometabolic risks that accompany childhood overweight and obesity.

The limitations of our study include the heterogeneity between the six study cohorts in age composition, sample size, and measurement methods for blood pressure, body composition, puberty assessment, and fasting insulin. Considering that the observed differences in adiposity and cardiometabolic characteristics were rather small, even when comparing between the upper and lower extremes of the genetic predisposition, no strong conclusion about the clinical significance of this genetic score can be drawn.

Genetic predisposition to increased insulin sensitivity and relatively higher fat storage on the hip compared to the waist leads to increased adiposity yet a favorable cardiometabolic profile in children and adolescents, indicative of a genetic basis of the controversial condition sometimes called "metabolically healthy obesity". Our findings provide novel insights into the link between adiposity and its complications in children and adolescents.

Acknowledgements We are extremely grateful to all the families who took part in ALSPAC, the midwives for their help in recruiting them, and the whole ALSPAC team, which includes interviewers, computer and laboratory technicians, clerical workers, research scientists, volunteers, managers, receptionists and nurses. We also especially want to express our thanks to the participating children and adolescents as well as their parents that were part of the YFS, TDCOB, STRIP, PANIC, and SKOT studies. We are also grateful to all members of these research teams for their skillful contributions in performing the studies.

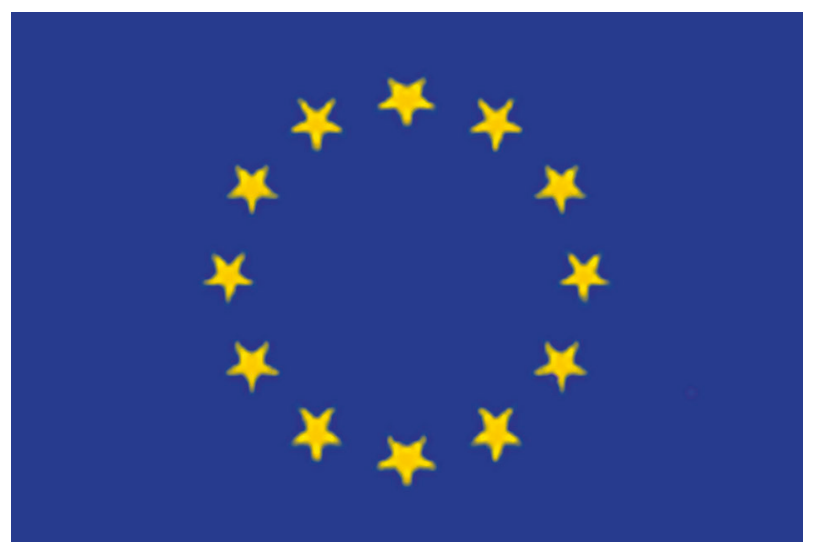

This project has received funding from the European Union's Horizon 2020 research and innovation programme under the Marie Sklodowska-Curie grant agreement no 796143. This project was also supported by North Savonia Regional Fund of Finnish Cultural Foundation, The Diabetes Research Foundation of Finland, Emil Aaltonen Foundation, Orion Research Foundation, the Danish Council for Independent Research (grant number DFF - 6110-00183), and the Novo Nordisk Foundation (grant numbers NNF17OC0026848, NNF15CC0018486, and NNF18CC0034900).

Funding The funders of the different studies are found in Supplementary Material. AV and TMS researched data, AV and TOK wrote the paper. Other coauthors reviewed/edited the paper and contributed to data collection. AV is the guarantor's of the article and takes responsibility for the contents of the article.

\section{Compliance with ethical standards}

Conflict of interest The authors declare that they have no conflict of interest.

Publisher's note: Springer Nature remains neutral with regard to jurisdictional claims in published maps and institutional affiliations. 


\section{References}

1. NCD Risk Factor Collaboration (NCD-RisC). Worldwide trends in body-mass index, underweight, overweight, and obesity from 1975 to 2016: a pooled analysis of 2416 population-based measurement studies in 128.9 million children, adolescents, and adults. Lancet. 2017;390:2627-42.

2. Poirier P, Giles TD, Bray GA, Hong Y, Stern JS, Pi-Sunyer FX. et al. Obesity and cardiovascular disease: pathophysiology, evaluation, and effect of weight loss. Arterioscler Thromb Vasc Biol. 2006;26:968-76.

3. Blüher S, Schwarz P. Metabolically healthy obesity from childhood to adulthood-does weight status alone matter? Metabolism. 2014;63:1084-92.

4. Lotta LA, Gulati P, Day FR, Payne F, Ongen H, van de Bunt M, et al. Integrative genomic analysis implicates limited peripheral adipose storage capacity in the pathogenesis of human insulin resistance. Nat Genet. 2017;49:17-26.

5. Shungin D, Winkler TW, Croteau-Chonka DC, Ferreira T, Locke $\mathrm{AE}$, Magi R, et al. New genetic loci link adipose and insulin biology to body fat distribution. Nature. 2015;518:187-96.

6. Virtue S, Vidal-Puig A. It's not how fat you are, it's what you do with it that counts. PLoS Biol. 2008;6:e237.

7. Stefan N, Schick F, Haring HUCauses. Characteristics, and consequences of metabolically unhealthy weight in humans. Cell Metab. 2017;26:292-300.

8. Li S, Chen W, Srinivasan SR, Xu J, Berenson GS. Relation of childhood obesity/cardiometabolic phenotypes to adult cardiometabolic profile: the Bogalusa Heart Study. Am J Epidemiol. 2012;176(Suppl 7):S142-9.

9. Boyd A, Golding J, Macleod J, Lawlor DA, Fraser A, Henderson J, et al. Cohort Profile: the 'children of the 90s'-the index offspring of the Avon Longitudinal Study of Parents and Children. Int J Epidemiol. 2013;42:111-27.

10. Åkerblom HK, Uhari M, Pesonen E, Dahl M, Kaprio EA, Nuutinen EM, et al. Cardiovascular risk in young Finns. Ann Med. 1991;23:35-39.

11. Holm JC, Gamborg M, Bille DS, Gr Nb K HN, Ward LC, Faerk J. Chronic care treatment of obese children and adolescents. Int $\mathbf{J}$ Pediatr Obes. 2011;6:188-96.

12. Simell O, Niinikoski H, Rönnemaa T, Raitakari OT, Lagström H, Laurinen M, et al. Cohort profile: the STRIP Study (Special Turku Coronary Risk Factor Intervention Project), an infancy-onset dietary and life-style intervention trial. Int J Epidemiol. 2009;38:650-5.

13. Eloranta AM, Lindi V, Schwab U, Kiiskinen S, Kalinkin M, Lakka HM, et al. Dietary factors and their associations with socioeconomic background in Finnish girls and boys 6-8 years of age: the PANIC Study. Eur J Clin Nutr. 2011;65:1211-8.

14. Andersen LB, Pipper CB, Trolle E, Bro R, Larnkjaer A, Carlsen EM, et al. Maternal obesity and offspring dietary patterns at 9 months of age. Eur J Clin Nutr. 2015;69:668-75.

15. Saari A, Sankilampi U, Hannila ML, Kiviniemi V, Kesseli K, Dunkel L. New Finnish growth references for children and adolescents aged 0 to 20 years: length/height-for-age, weight-forlength/height, and body mass index-for-age. Ann Med. 2011;43: $235-48$.

16. Nysom K, Molgaard C, Hutchings B, Michaelsen KF. Body mass index of 0 to 45-y-old Danes: reference values and comparison with published European reference values. Int J Obes Relat Metab Disord. 2001;25:177-84.

17. Cole TJ, Freeman JV, Preece MA. Body mass index reference curves for the UK, 1990. Arch Dis Child. 1995;73:25-29.

18. Cole TJ, Lobstein T. Extended international (IOTF) body mass index cut-offs for thinness, overweight and obesity. Pediatr Obes. 2012;7:284-94.
19. Marshall WA, Tanner JM. Variations in pattern of pubertal changes in girls. Arch Dis Child. 1969;44:291-303.

20. Marshall WA, Tanner JM. Variations in the pattern of pubertal changes in boys. Arch Dis Child. 1970;45:13-23.

21. Falaschetti E, Hingorani AD, Jones A, Charakida M, Finer N, Whincup $\mathrm{P}$, et al. Adiposity and cardiovascular risk factors in a large contemporary population of pre-pubertal children. Eur Heart J. 2010;31:3063-72.

22. Raitakari OT, Juonala M, Rönnemaa T, Keltikangas-Järvinen L, Räsänen L, Pietikainen M, et al. Cohort profile: the cardiovascular risk in Young Finns Study. Int J Epidemiol. 2008;37:1220-6.

23. Fonvig CE, Chabanova E, Ohrt JD, Nielsen LA, Pedersen O, Hansen T.et al. Multidisciplinary care of obese children and adolescents for one year reduces ectopic fat content in liver and skeletal muscle. BMC Pediatr. 2015;15:196-015-0513-6.

24. Viitasalo A, Laaksonen DE, Lindi V, Eloranta AM, Jääskelainen J, Tompuri T, et al. Clustering of metabolic risk factors is associated with high-normal levels of liver enzymes among 6- to 8-year-old Children: the PANIC study. Metab Syndr Relat Disord. 2012;10:337-43.

25. Madsen AL, Schack-Nielsen L, Larnkjaer A, Molgaard C, Michaelsen KF. Determinants of blood glucose and insulin in healthy 9-month-old term Danish infants; the SKOT cohort. Diabet Med. 2010;27:1350-7.

26. Johnson T. Efficient calculation for multi-snp genetic scores. Poster presentation, American Society of Human Genetics Annual Meeting. San Francisco, CA. 2012.

27. Felix JF, Bradfield JP, Monnereau C, van der Valk RJ, Stergiakouli E, Chesi A, et al. Genome-wide association analysis identifies three new susceptibility loci for childhood body mass index. Hum Mol Genet. 2016;25:389-403.

28. Casale FP, Rakitsch B, Lippert C, Stegle O. Efficient set tests for the genetic analysis of correlated traits. Nat Methods. 2015;12:755-8.

29. Winkler TW, Justice AE, Graff M, Barata L, Feitosa MF, Chu S, et al. Correction: the influence of age and sex on genetic associations with adult body size and shape: a large-scale genomewide interaction study. PLoS Genet. 2016;12:e1006166.

30. Graae AS, Hollensted M, Kloppenborg JT, Mahendran Y, Schnurr TM, Appel EVR, et al. An adult-based insulin resistance genetic score associates with insulin resistance, metabolic traits and altered fat distribution in Danish children and adolescents who are overweight or obese. Diabetologia. 2018;61:1769-79.

31. Jolliffe CJ, Janssen I. Development of age-specific adolescent metabolic syndrome criteria that are linked to the Adult Treatment Panel III and International Diabetes Federation criteria. J Am Coll Cardiol. 2007;49:891-8.

32. Prince RL, Kuk JL, Ambler KA, Dhaliwal J, Ball GD. Predictors of metabolically healthy obesity in children. Diabetes Care. 2014;37:1462-8.

33. Li L, Yin J, Cheng H, Wang Y, Gao S, Li M, et al. Identification of genetic and environmental factors predicting metabolically healthy obesity in children: data from the BCAMS study. J Clin Endocrinol Metab. 2016;101:1816-25.

34. Carobbio S, Pellegrinelli V, Vidal-Puig A. Adipose tissue function and expandability as determinants of lipotoxicity and the metabolic syndrome. Adv Exp Med Biol. 2017;960:161-96.

35. Karelis AD. Metabolically healthy but obese individuals. Lancet. 2008;372:1281-3.

36. Kantartzis K, Machann J, Schick F, Rittig K, Machicao F, Fritsche $\mathrm{A}$, et al. Effects of a lifestyle intervention in metabolically benign and malign obesity. Diabetologia. 2011;54:864-8.

37. Heymsfield SB, Wadden TA. Mechanisms, pathophysiology, and management of obesity. N Engl J Med. 2017;376:1492.

38. Kelsey MM, Zeitler PS. Insulin resistance of puberty. Curr Diab Rep. 2016;16:64-016-0751-5. 
39. Reinehr T, Wolters B, Knop C, Lass N, Holl RW. Strong effect of pubertal status on metabolic health in obese children: a longitudinal study. J Clin Endocrinol Metab. 2015;100:301-8.
40. Knowles JW, Ashley EA. Cardiovascular disease: the rise of the genetic score. PLoS Med. 2018;15:e1002546.

\section{Affiliations}

Anna Viitasalo ${ }^{1}$ Theresia M. Schnurr $\mathbb{D}^{1} \cdot$ Niina Pitkänen $\mathbb{D}^{2} \cdot$ Mette Hollensted $^{1} \cdot$ Tenna R. H. Nielsen $^{3,4}$. Katja Pahkala ${ }^{2,5} \cdot$ Niina Lintu $^{6}$. Mads V. Lind $\mathbb{D}^{7} \cdot$ Mustafa Atalay $^{6} \cdot$ Christine Frithioff-Bøjsøe $^{1,3}$.

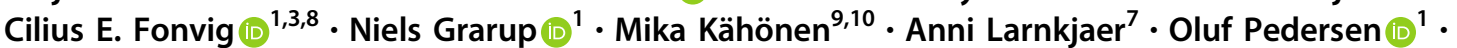
Jens-Christian Holm ${ }^{1,3,11} \cdot$ Kim F. Michaelsen ${ }^{7} \cdot$ Timo A. Lakka $^{6,12,13} \cdot$ Terho Lehtimäki $^{10,14} \cdot$ Olli Raitakari $^{2,15}$. Torben Hansen ${ }^{1} \cdot$ Tuomas O. Kilpeläinen ${ }^{1}$

1 Novo Nordisk Foundation Center for Basic Metabolic Research, Faculty of Health and Medical Sciences, University of Copenhagen, Copenhagen, Denmark

2 Research Centre of Applied and Preventive Cardiovascular Medicine, University of Turku, Turku, Finland

3 The Children's Obesity Clinic, Department of Pediatrics, Copenhagen University Hospital Holbæk, Holbæk, Denmark

4 Department of Pediatrics, Copenhagen University Hospital Hvidovre, Hvidovre, Denmark

5 Paavo Nurmi Centre, Sports and Exercise Medicine Unit, Department of Physical Activity and Health, University of Turku, Turku, Finland

6 Institute of Biomedicine, University of Eastern Finland, Kuopio, Finland

7 Department of Nutrition, Exercise and Sports, Faculty of Science, University of Copenhagen, Copenhagen, Denmark

8 The Hans Christian Andersen Children's Hospital, Odense
University Hospital, Odense, Denmark

9 Department of Clinical Physiology, Tampere University Hospital, Tampere, Finland

10 Faculty of Medicine and Health Technology, Finnish Cardiovascular Research Center, Tampere University, Tampere, Finland

11 University of Copenhagen, Faculty of Health and Medical Sciences, Copenhagen, Denmark

12 Department of Clinical Physiology and Nuclear Medicine, Kuopio University Hospital, Kuopio, Finland

13 Kuopio Research Institute of Exercise Medicine, Kuopio, Finland

14 Department of Clinical Chemistry, Fimlab Laboratories, Tampere, Finland

15 Department of Clinical Physiology and Nuclear Medicine, Turku University Hospital, Turku, Finland 